\title{
Empirical Derivation and Validation of a Wandering Typology
}

\author{
Donna L. Algase, PhD, * Cathy Antonakos, PhD, ${ }^{*}$ Elizabeth R. A. Beattie, PhD, ${ }^{\dagger}$ \\ Cynthia A. Beel-Bates, PhD, ${ }^{\mathcal{S}}$ and Lan Yao, PhD*
}

OBJECTIVES: To develop and validate a wandering typology.

DESIGN: Cross-sectional, correlational descriptive design. SETTING:Twenty-two nursing homes and six assisted living facilities.

PARTICIPANTS: One hundred forty-two residents with dementia who spoke English, met Diagnostic and Statistical Manual for Mental Disorders, Fourth Edition, criteria for dementia, scored less than 24 on the Mini-Mental State Examination (MMSE), were ambulatory (with or without assistive device), and maintained a stable regime of psychotropic medications were studied.

MEASUREMENTS: Data on wandering were collected using direct observations, plotted serially according to rate and duration to yield 21 parameters, and reduced through factor analysis to four components: high rate, high duration, low to moderate rate and duration, and time of day. Other measures included the MMSE, Minimum Data Set 2.0 mobility items, Cumulative Illness Rating Scale-Geriatric, and tympanic body temperature readings.

RESULTS: Three groups of wanderers were identified through cluster analysis: classic, moderate, and subclinical. MMSE, mobility, and cardiac and upper and lower gastrointestinal problems differed between groups of wanderers and in comparison with nonwanderers.

CONCLUSION: Results have implications for improving identification of wanderers and treatment of possible contributing factors. J Am Geriatr Soc 57:2037-2045, 2009.

Key words: wandering; typology; observation; dementia; cluster analysis

From the *School of Nursing, University of Michigan, Ann Arbor, Michigan; 'School of Nursing, Queensland University of Technology, Brisbane, Queensland, Australia; and ${ }^{\S}$ School of Nursing, Grand Valley State University, Grand Rapids, Michigan.

Address correspondence to Donna L. Algase, University of Michigan School of Nursing, 400 N. Ingalls Rd., Ann Arbor, MI 48109. E-mail: dalgase@umich.edu DOI: $10.1111 /$ j.1532-5415.2009.02491.x
W andering is common in nursing home and assisted living residents with dementia. Sometimes regarded as benign, wandering poses significant risks for malnutrition and dehydration, falls and other injuries, elopements, getting lost, and early mortality ${ }^{1-5}$ and often incurs extraordinary costs for providing appropriate care and resolving legal claims. ${ }^{6-8}$ Despite risks and costs, wandering has received scant scientific attention. Little of its etiology is understood; empirically tested interventions are few and marginally effective in ameliorating the behavior.

A major reason for limited progress in understanding wandering and its negative consequences is that the behavior itself has not been well characterized. A recent investigation found that few studies have described actual wandering and that methods for doing so varied considerably across these studies, inhibiting integration of findings. ${ }^{9}$ In efforts to better characterize wandering, several typologies have been proposed, ${ }^{10-12}$ although they generally lack empirical grounding. Only an empirically derived typology developed previously, ${ }^{13}$ which is focused solely on geographic patterns (lapping, pacing, random, direct) of individual ambulation episodes, has influenced further research. Subsequent studies ${ }^{14,15}$ have validated these patterns, demonstrating that random is most common and that affected individuals often exhibit more than one pattern.

Although useful, patterns do not differentiate between wanderers. Descriptions of wandering at the level of persons can be the basis for deriving better understanding of etiologies for wandering, evaluating potential genetic contributions to wandering, and targeting interventions with greater accuracy. The primary aim of this study was to develop and validate a typology of wandering at the person, rather than episode, level. This typology is derived using a new set of parameters for assessing wandering based on an individual's variation in rate and duration of wandering episodes over a series of daytime observations.

This study was conducted within the context of the Need-driven Dementia-compromised Behavior (NDB) model. ${ }^{16}$ Accordingly, a set of stable background factors (e.g., cognitive impairment, circadian rhythm, mobility, age, sex, general health) are postulated to predict individuals at risk for wandering and other dementia-related 
behaviors (e.g., aggression, problematic vocalizations); another set of more-dynamic proximal factors (e.g., physiological and psychological needs, features of the social and physical environment) constitute the context within which wandering is expressed in those at risk. In this study validation variables were selected to evaluate differences between subtypes of wanderers. Thus, several background factors, measured at the person level, were examined: cognitive impairment, measured according to Mini-Mental State Examination (MMSE) score; circadian rhythm, evaluated according to tympanic temperature readings; mobility, obtained from Minimum Data Set mobility items; age and sex, derived from chart review; and general health, assessed by nurse practitioners according to 14 organ systems delineated in the Cumulative Illness Rating Scale - Geriatric version (CIRS-G). Proximal factors were not examined because of their postulated transient and dynamic effects on behavior and their measurement at the observation period level and were controlled through randomization of observation periods.

\section{METHODS}

\section{Design}

A descriptive, cross-sectional correlational design was used within the context of a larger study testing the NDB model. ${ }^{16}$ In the parent study, ambulatory individuals $(\mathrm{N}=181)$ with dementia from 22 nursing homes (NHs) and six assisted living facilities (ALFs) were videotaped in their natural surroundings, and tapes were coded for individual wandering episodes. In the present analysis, parameters describing individual variation in rate and duration of wandering, drawn from the set of observations, were used to derive a wandering typology. Institutional review boards of two participating universities approved study procedures; each study site received a single federal project assurance. Proxies provided consent for participants with dementia; participants provided assent for participation at each observation.

\section{Sample}

To ensure that each participant had a sufficient number of observations to capture variation in wandering rate and duration, those who completed 10 or more of 12 scheduled observations in the parent project were chosen for secondary analysis. Participants $(\mathrm{N}=142$, or $78.5 \%$ of the parent sample) fulfilled the following inclusion criteria: spoke English, met Diagnostic and Statistical Manual of Mental Disorders, Fourth Edition, criteria for medical diagnosis of dementia, scored less than 24 on the MMSE, were ambulatory (with or without assistive device), and maintained a stable regime of psychotropic medications, if any, over 30 days before and during observations. Participants did not differ from others in the parent study in terms of sex, age, MMSE score, mobility, or residence (NH vs ALF).

The overall sample was $76.1 \%$ female $(\mathrm{n}=108)$ with a mean age standard deviation of $83.7 \pm 6.4$ (range 68-102). Mean MMSE score $(\mathrm{n}=96)$ was $10.5 \pm 6.2$ (range 0-23). Ninety-four participants $(66.2 \%)$ were independently mobile; 46 (32.4\%) others required some assistance (e.g., cane, walker) to ambulate, data were missing on two.

\section{Measures for Dependent Variables}

Wandering was quantified based on four scores derived from a principal components analysis (PCA): low to moderate wandering output, high wandering durations, high wandering rates, and time of day. In the parent study, each participant was videotaped for twelve 20 -minute periods occurring once per hour between 8:00 a.m. and 8:00 p.m.; periods were randomly distributed to the first or second half of the hour and over 2 days, separated by a 2-day hiatus. Recorded ambulation episodes were coded for wandering behavior using Noldus Observer 5.0 software (Noldus Information Technology Inc., Leesburg, VA). Wandering was operationalized according to geographic patterns of lapping, pacing, and random walking; direct walking (paths leading to a destination without deviation) was considered not to be wandering. ${ }^{13} \mathrm{~A}$ full description of coding procedures has been published elsewhere. ${ }^{17}$

In an earlier analysis using the parent data, ${ }^{9} 21 \mathrm{pa}-$ rameters of wandering were derived from 44 known wanderers who had a complete set of 12 observations. Wandering rate and duration data from each observation were plotted according to participant to provide a serial view; the distribution of data points above and below a participant's median rate and duration values was used to determine their values on each parameter. Examples of parameters are mean hourly rate, mean hourly duration, percentage of hours with any wandering, peak rate, and maximum hourly duration. (See Table 1 for full list.) These analytical procedures were extended to generate values for parameters for all participants in the study; results were converted to z-scores and used as inputs for a PCA and in computation of component scores to be used in a subsequent cluster analysis for deriving the wandering typology. Results of the PCA are shown in Table 1. Component scores were the linear combination of a participant's values on all parameters, weighted by their loadings on each component.

Ratings of wandering status by nursing staff were also obtained on a 4-point ordinal scale: from "definitely not a wanderer" to "yes, a problematic wanderer." Such ratings correlate highly with observed wandering. ${ }^{18}$

\section{Measures for Independent (Validation) Variables}

Validation variables were chosen from among background factors of the NDB model and included cognitive impairment, mobility, general health, circadian rhythm disturbances, age, and sex. ${ }^{16}$

Cognitive impairment was assessed using the MMSE, ${ }^{19}$ a valid and reliable tool ${ }^{18-21}$ widely used in research and standard dementia assessments. The MMSE assesses orientation, registration, attention, calculation, recall, language, and construction tasks to yield a global performance score between 0 (severely impaired) and 30 (no impairment). To minimize missing data and address floor effects of the MMSE, 37 participants who were too impaired to complete testing were assigned a score of -1 , consistent with previous studies. ${ }^{22,23} \mathrm{~A}$ Ph.D. research nurse trained by a consulting neuropsychologist in administration of the MMSE collected MMSE data.

Mobility was categorized based on the Minimum Data Set (MDS 2.0). Those whose ratings indicated independent 
Table 1. Component Loadings for 20 Wandering Parameters for a Principal Component Analysis (PCA) with Orthogonal Rotation $(\mathrm{N}=123)$

\begin{tabular}{|c|c|c|c|c|}
\hline$\%$ of observations duration $>0$ and $<15$ & 0.426 & & & \\
\hline$\%$ of hours with any nonwandering locomotion & 0.404 & & & \\
\hline$\%$ hours with any wandering episode & 0.417 & & & \\
\hline Mean hourly rate for nonwandering locomotion & 0.330 & & & \\
\hline$\%$ of observations rate $=0$ & -0.331 & & & \\
\hline Mean hourly duration & & 0.523 & & \\
\hline Mean duration per episode & & 0.438 & & \\
\hline$\%$ observations duration $>15 \mathrm{~min} / \mathrm{h}$ & & 0.406 & & \\
\hline Max. peak of mean hourly duration & & 0.404 & & \\
\hline Mean hourly duration category $(0,>0-15)>15 \mathrm{~min} / \mathrm{h}$ & & 0.282 & & \\
\hline Mean hourly rate category $(0,1-6,>6$ times/h) & & & 0.358 & \\
\hline Time of max. rate & & & & 0.497 \\
\hline Maximum rate category: $8 a-12 n, 12 n-4 p, 4 p-8 p$ & & & & 0.504 \\
\hline Time of maximum duration & & & & 0.492 \\
\hline Maximum duration category: $8 a-12 n, 12 n-4 p, 4 p-8 p$ & & & & 0.500 \\
\hline Eigen value & 8.561 & 3.669 & 3.011 & 1.610 \\
\hline$\%$ explained variance & 25.42 & 21.25 & 20.48 & 14.45 \\
\hline
\end{tabular}

performance on all items in Section G (bed mobility, transfer, walk in room, walk in corridor, locomotion on and off unit) were designated independent or, if not independent in performing any item, as assisted. Reliability and validity of the MDS is acceptable for assessing a wide range of resident conditions. ${ }^{24-26}$

General health was assessed using the CIRS-G. The CIRS-G, which has established validity and reliability, ${ }^{27}$ estimates comorbidity based on physician or nurse practitioner ratings of presence and severity of disease for 14 organ systems, with 0 indicating no disease present and 4 indicating severe disease. An average score across systems was calculated, with a possible range of 0 to 4 , higher scores indicating poorer health.

Circadian rhythm was assessed according to tympanic temperature readings at 3-hour intervals over 36 hours FirstTemp Genius infrared tympanic thermometer (Covidien, Mansfield, MA). ${ }^{28}$ Correlations between tympanic temperatures using the FirstTemp Genius and core body temperature ranges from 0.72 to $0.90 .{ }^{29-31}$ The tympanic temperature method was chosen, because it was reliable and noninvasive and overcame difficulty in gaining cooperation of dementia patients in keeping their mouths closed with the probe under their tongues. Two parameters of circadian rhythm, acrophase and amplitude, which were determined using sigmoidally transformed cosine waves, ${ }^{32}$ were used in analyses. Acrophase is the time of peak of the fitted curve; amplitude is the difference between the minimum and maximum of the fitted curve.

Age and sex were extracted from participants' medical records.

\section{Procedures}

Potential participants were recruited using surrogate informed consent from legally authorized representatives; assent of participants was assessed with each data collection episode. The MMSE was administered first to validate eligibility for the study. Participants were offered food, water, and an opportunity to use the restroom before testing. Testing was conducted in a quiet room without interruptions. The research nurse briefly socialized with each participant to establish rapport and create a comfortable setting before testing.

Research geriatric nurse practitioners (GNPs) made CIRS-G ratings based on a complete physical examination and review of medical records for medical diagnoses, current medications (standing and as needed), and laboratory values. Written informed consent documentation to access chart data was shown to the MDS coordinator at each nursing home. Mobility status was determined from the most recent (within 3 months) MDS 2.0 record in the participant's chart; in ALFs, where the MDS is not required, research GNPs completed MDS mobility items for participants. Next, temperature data were obtained every 3 hours over a 36-hour period beginning at 6:00 p.m., with two tympanic temperatures taken at each reading, including while participants were asleep. Before initial temperature measurement, the ear canal was inspected for impacted wax, fluid, or blood. If present, measurement was taken in the other ear. If the resident wore a hearing aid, it was removed for 10 minutes before temperature assessment. Within 30 seconds of applying a new cover to the probe, the 
temperature was taken by retracting the tragus, inserting the thermometer into the ear canal parallel to the jaw line to seal the opening, and pressing the scan button. Within 2 seconds, the temperature and the word "done" appeared in the thermometer's display window. A second temperature was taken 2 minutes later following the same procedure. The first and second temperatures were recorded, and the highest value was used in analyses, because lower readings most often result from improper probe placement. Calibration of thermometers was conducted every 2 months, with calibration accuracy limits based on American Society for Testing and Materials Standard E1112-86. Observations of wandering were made during the week after data collection for measures of independent variables.

\section{Data Analysis}

All analyses were conducted using Stata 9.2 (StataCorp., College Station, TX). PCA with varimax rotation was used to reduce the set of 21 wandering parameters to a minimum number of components and to maximize explained variance. The number of components in the final solution was determined by applying the Kaiser criterion (eigenvalue $>1$ ) and retaining components that had a minimum of three variables with loadings more than 0.3 . Component scores were generated from the rotated solution using the predictcommand postestimation.

Cluster analysis was used to identify groups of individuals with similar combinations of loadings on component scores. The four component scores generated from the PCA were used as variables in the cluster analysis. Ward's linkage, which minimizes within-cluster variance, was used to form clusters. Squared Euclidean distances were used as input; squared Euclidean distance represents the difference between two cases across the four component scores.

The number of clusters chosen for the final solution was determined by examining the dendrogram and using the Calinski-Harabasz pseudo-F stopping rule index. A dendrogram provides a graphical representation of the clusters of cases. To interpret the graph, the vertical height of lines separating clusters were focused on as confirmation of a three-cluster solution; longer vertical lines indicate moredistinct clusters. The number of cases was also examined, avoiding solutions that yielded few cases in any one cluster.

To validate the three-cluster solution, analyses of variance (ANOVAs) and chi-square tests of association were conducted to compare clusters and the residual group of unclustered cases (nonwanderers). Variables used for cluster comparisons were not used to generate the cluster solution. They included age, sex, MMSE score, mobility,
CIRS-G score, and circadian rhythm acrophase and amplitude.

\section{RESULTS}

\section{Cluster Analysis}

Analysis resulted in three clusters and left a group of 19 participants unassigned to any cluster. The three clusters had 55, 14, and 54 cases. The Calinski-Harabasz pseudo-F stopping rule index associated with this solution was 46.9 . Larger values of the pseudo-F indicate distinct structure (large between-group variance relative to within-group variance).

Mean components scores and comparative rankings are shown for each cluster in Table 2.

Scores had the greatest spread on the high duration component (absolute difference $=5.72$ between the highest and lowest mean $z$-scores across clusters) and the lowest on time of day (absolute difference $=0.28$ ), indicating that clusters are most distinct on the basis of high wandering duration and least distinct on time of day for highest peak for wandering rate and duration.

Cluster A contains individuals $(n=14,9.9 \%$ of the sample) with substantially higher component scores on high wandering duration and rate components than other clusters. This cluster also had the lowest mean component score for time of day, indicating that, on average, their highest hourly wandering rate and duration occurred earlier in the day than in other clusters. Individuals in Cluster $\mathrm{B}(\mathrm{n}=55$, $38.7 \%$ ) had middle scores on high rate, high duration, and moderate movement components and highest scores on the time of day component, indicating low to moderate amount of wandering with highest hourly rate and duration occurring somewhat later in the day and at lower values than Cluster A. Cluster C ( $\mathrm{n}=54,38.0 \%)$ had the lowest scores on three components (high rate, high duration, and low to moderate movement) and was close to Cluster A on the time of day component, indicating the least amount of overall wandering, with their highest hourly rate and duration occurring relatively early in the day. The group of unclustered participants $(\mathrm{n}=19,13.4 \%)$ were those who displayed no wandering during any observation period and are referred to hereafter as nonwanderers.

For ease in relating components to observed wandering, actual values (rather than $z$-scores) for wandering parameters, grouped according to component on which they had the highest loading, are shown for each cluster (Table 3). Nonwanderers thus had scores of 0 on all parameters except those for direct (nonwandering) ambu-

Table 2. Average Component Scores and Their Comparative Rankings According to Cluster $(\mathrm{N}=142)$

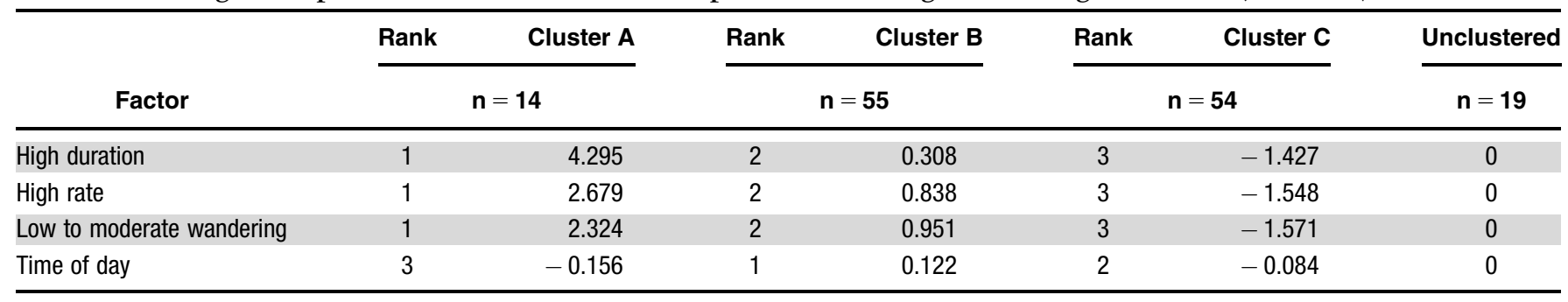




\begin{tabular}{|c|c|c|c|c|}
\hline & Cluster A & Cluster B & Cluster C & Nonwanderers \\
\hline Parameter & \multicolumn{4}{|c|}{ M (SD) } \\
\hline \multicolumn{5}{|l|}{ Wandering parameter, mean $\pm \mathrm{SD}$} \\
\hline \multicolumn{5}{|l|}{ High duration } \\
\hline Mean duration per episode, minutes & $1.5 \pm 1.2$ & $0.5 \pm 0.5$ & $0.1 \pm 0.1$ & 0 \\
\hline Mean hourly duration, minutes & $15.1 \pm 5.8$ & $4.3 \pm 2.6$ & $0.9 \pm 0.7$ & 0 \\
\hline Maximum peak, mean hourly duration, minutes & $43.3 \pm 10.6$ & $20.96 \pm 12.5$ & $6.3 \pm 4.6$ & 0 \\
\hline$\%$ observations duration $>15 \mathrm{~min} / \mathrm{h}$ & $40.1 \pm 15.2$ & $9.1 \pm 8.8$ & $0.3 \pm 0.0$ & 0 \\
\hline \multicolumn{5}{|l|}{ High rate } \\
\hline Mean hourly rate & $9.3 \pm 5.1$ & $4.3 \pm 2.8$ & $0.8 \pm 0.8$ & 0 \\
\hline Maximum rate per hours & $29.6 \pm 14.3$ & $20.0 \pm 11.4$ & $5.5 \pm 5.3$ & 0 \\
\hline$\%$ of observations rate $>6$ times $/ \mathrm{h}$ & $41.2 \pm 18.6$ & $22.1 \pm 15.4$ & $2.7 \pm 0.1$ & 0 \\
\hline \multicolumn{5}{|l|}{ Low to moderate movement } \\
\hline$\%$ hours with any wandering & $74.9 \pm 11.5$ & $50.1 \pm 14.1$ & $24.9 \pm 12.8$ & 0 \\
\hline$\%$ of observations rate and duration $=0$ & $25.4 \pm 11.5$ & $49.9 \pm 14.1$ & $75.1 \pm 12.8$ & $100.0 \pm 0.0$ \\
\hline$\%$ of observations duration $>0$ and $<15 \mathrm{~min} / \mathrm{h}$ & $34.5 \pm 13.1$ & $41.0 \pm 18.7$ & $24.6 \pm 12.7$ & 0 \\
\hline$\%$ of observations rate $=1-6$ times $/ \mathrm{h}$ & $33.4 \pm 19.0$ & $28.0 \pm 18.7$ & $22.2 \pm 12.7$ & 0 \\
\hline \multicolumn{5}{|l|}{ Time of day } \\
\hline Time of maximum rate* & $1,442 \pm 3.1$ & $1,457 \pm 3.4$ & $1,506 \pm 3.3$ & 0 \\
\hline Time of maximum duration* & $1,500 \pm 2.8$ & $1,510 \pm 3.6$ & $1,432 \pm 3.1$ & 0 \\
\hline \multicolumn{5}{|l|}{ Nonwandering parameter } \\
\hline$\%$ of hours with direct locomotion, mean $\pm \mathrm{SD}$ & $42.6 \pm 18.5$ & $41.2 \pm 16.7$ & $25.1 \pm 16.0$ & $31.1 \pm 19.4$ \\
\hline Mean hourly duration, direct, mean \pm SD & $0.2 \pm 0.2$ & $0.2 \pm 0.1$ & $0.1 \pm 0.1$ & $0.06 \pm 0.11$ \\
\hline Mean hourly rate, direct, mean \pm SD & $4.4 \pm 2.4$ & $3.3 \pm 2.8$ & $1.7 \pm 1.4$ & $0.51 \pm 0.57$ \\
\hline \multicolumn{5}{|l|}{ Validation measure } \\
\hline Age, mean $\pm S D$ & $83.0 \pm 7.8$ & $82.5 \pm 6.4$ & $85.2 \pm 6.0$ & $83.7 \pm 6.3$ \\
\hline Mini-Mental State Examination score, mean \pm SD (range 1-23) & $2.5 \pm 4.9$ & $6.5 \pm 7.3$ & $9.4 \pm 6.9$ & $7.3 \pm 7.4$ \\
\hline Cumulative IIIness Rating Scale-Geriatric version score, mean $\pm S D$ (range 0-1) & $0.8 \pm 0.2$ & $0.6 \pm 0.2$ & $0.7 \pm 0.2$ & $0.73 \pm 0.28$ \\
\hline Acrophase, mean \pm SD (range 0800-1759)* & $1,535 \pm 3.9$ & $1,425 \pm 5.8$ & $1,545 \pm 4.9$ & $1,542 \pm 5.82$ \\
\hline Amplitude, ${ }^{\circ} \mathrm{C}$ & $1.1 \pm 0.5$ & $1.2 \pm 1.5$ & $1.3 \pm 1.5$ & $1.0 \pm 0.4$ \\
\hline Female, \% & 78.6 & 78.2 & 78.0 & 68.4 \\
\hline Independent in mobility, \% & 92.9 & 64.8 & 67.9 & 52.6 \\
\hline
\end{tabular}

* Mean and range in military time, standard deviation (SD) in decimal hours.

lation. Graphs of hourly rate and duration across observation periods (Figure 1) are displayed for one participant characteristic of each cluster.

\section{Cluster Validation}

One-way ANOVAs were used to assess differences between clusters and nonwanderers on validation variables with continuous data. No differences were revealed for age (degrees of freedom $(d f)(3,138) F=1.65 ; P=.18)$ or either parameter of circadian rhythm (amplitude, $d f(3,133)$ $F=0.38 ; P=.77$ and acrophase: $d f(3,133) F=0.60$; $P=.62)$, but significant differences were found for cognitive impairment $(d f(3,129) F=3.60 ; P<.015)$ and general health $(d f(3,133) F=3.59$; $P=.01)$. Cluster A wanderers had the lowest mean MMSE score $(2.5 \pm 4.9)$ and Cluster B wanderers the highest $(9.41 \pm 6.9)$. Cluster $\mathrm{B}$ wanderers also had the best general health (CIRS-G $0.61 \pm 0.22$ ), whereas all others had similar health scores - between 0.73 for nonwanderers and 0.75 for Cluster A wanderers.
Chi-square $\left(\chi^{2}\right)$ tests were used to assess association between categorical variables and all clusters and the nonwanderers. No association was found for sex (df (3) $\left.\chi^{2}=0.79, P=.85\right)$, but the association for mobility approached significance $\left(d f(3) \chi^{2}=6.16, P=.10\right) ; 13$ of 14 wanderers $(92.9 \%)$ in Cluster A were independent in mobility, whereas the percentages in Cluster B, Cluster C, and nonwanderers were $64.8 \%, 67.9 \%$, and $52.6 \%$, respectively. Last, the association between staff ratings of wandering status and wandering clusters and nonwanderers was also evaluated using chi-square. Removing the ambiguous rating of "sometimes a wanderer" and collapsing both yes categories (indicating presence of problematic and nonproblematic wandering), a highly significant association was found ( $d f(3) \chi^{2}=11.48 ; P=.009$ ). The pattern of misclassifications was also noteworthy. Nursing staff ratings corresponded with cluster membership for $80 \%$ of wanderers in Cluster A and $78 \%$ in Cluster B, whereas ratings corresponded for only $43 \%$ of wanderers in Cluster C and $64 \%$ of nonwanderers. The association was nearly significant when all cases with a staff rating $(\mathrm{n}=111)$ were 

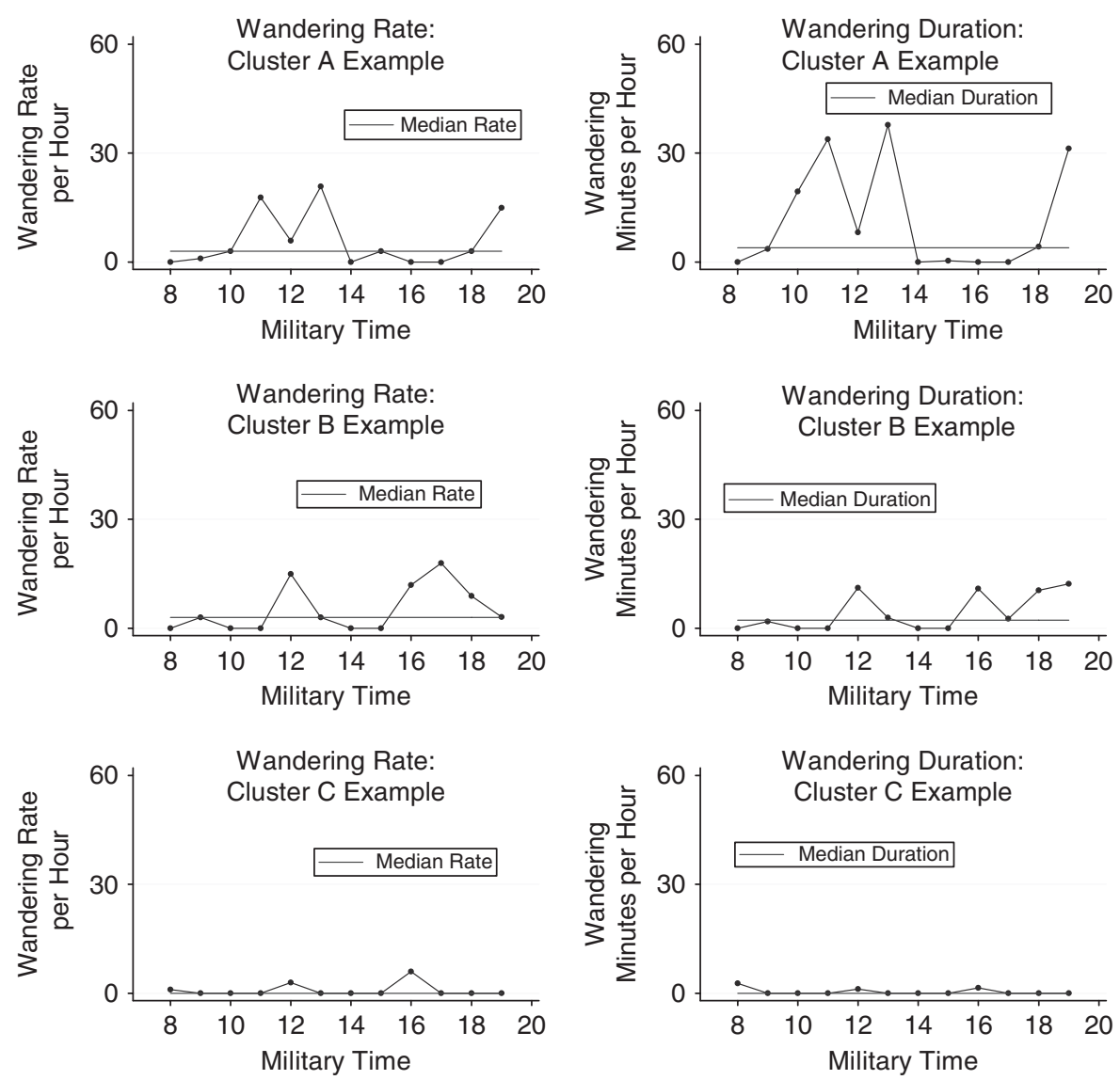

Figure 1. Case examples of hourly distributions for wandering rate and duration according to cluster.

used and both yes categories (problematic and nonproblematic) remained separate $\left(d f(9) ; \chi^{2}=15.67 ; P=.07\right)$.

\section{Post Hoc Analyses}

Given that wanderers have been attributed with better health than nonwanderers, ${ }^{33}$ the finding that only Cluster B displayed better health was further explored. One-way ANOVAs were performed to compare clusters on ratings for each CIRS-G organ system, using a Bonferronicorrected alpha of 0.0056 to accommodate multiple tests (Bonferroni corrected alpha $=\mathrm{alpha} / \mathrm{n}$ of tests or $.05 /$ $9=0.0056$ ). Figure 2 displays means for all clusters and nonwanderers on systems in which significant and nearsignificant differences were found (heart, $d f(3,133)$ $F=4.73, P=.004$; upper gastrointestinal and nutritional status, $d f(3,133) F=2.56, P=.06$; and lower gastrointestinal, $d f(3,133) F=4.06, P=.009)$. Nonsignificant findings for musculoskeletal or integumentary, neurological, and psychiatric illnesses are noteworthy.

Finally, variables that were significant or trending to significance in differentiating between clusters were used together in a multinomial logistic regression to predict probability of cluster membership compared with nonwanderers $(\mathrm{n}=129)$. Overall results were highly significant (likelihood ratio $\left.\chi^{2}(15)=43.75, P<.001\right)$, indicating a good fit to the model. Two predictors were significant for Cluster A: mobility (coefficient $=2.38, P=.048$ ) and cognitive impairment (coefficient $=-0.41, P=.047$ ); CIRS-G heart rating ap- proached significance (coefficient $=-0.803, P=.13$ ). Two other predictors were significant for Cluster B: upper gastrointestinal and nutritional status (coefficient $=0.807$, $P=.02$ ) and lower gastrointestinal (coefficient $=0.881$, $P=.02$ ). No predictors were significant for Cluster C.

\section{DISCUSSON}

This study resulted in an empirically derived typology of wanderers based on variation in wandering rate and duration over the course of daytime hours. This typology pro-

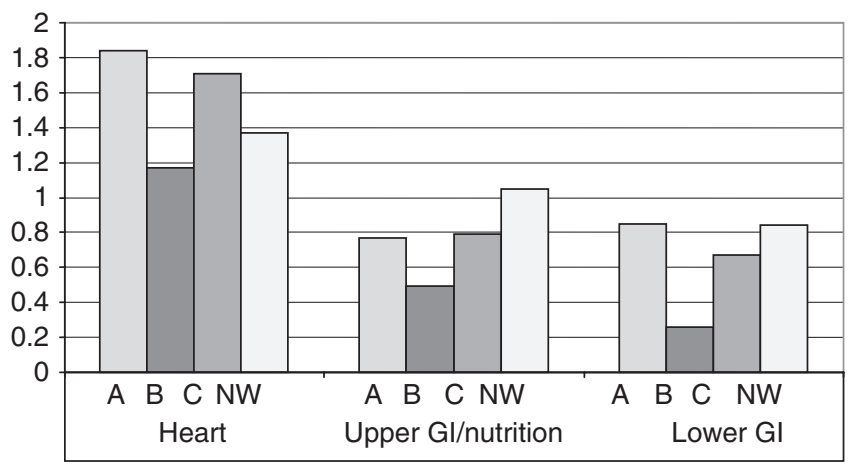

Figure 2. Item means (scale 0-4) for Cumulative Illness Rating Scale-Geriatric version systems (heart, upper gastrointestinal (GI) and nutrition, lower GI) according to cluster (A, B, C, and nonwanderers). 
vides a means to better characterize wanderers in regard to their overall expression or possible phenotype of wandering and to examine variation in potential etiological factors for the behavior. Furthermore, some background factors from the NDB model were useful in demonstrating cluster-level differences between types of wanderers and in comparison with nonwanderers. Consequently, this study extends descriptive information about wandering beyond its basic geographic patterns, as revealed in the typology developed previously. ${ }^{13}$

Analyses revealed three distinct clusters of wanderers that each differed significantly from nonwanderers overall. Component scores, values of key wandering parameters, and results of multinomial logistic regression characterize clusters. Accordingly, Cluster A members, termed classic wanderers, exhibited the most wandering according to rate and duration. They wandered during a greater percentage of observations, as well as more within an observation period, than any other group. Classic wanderers were more likely to have their highest hourly wandering rate and duration occur slightly earlier in the day, on average just before 3:00 p.m. Overall differences between groups were small in terms of time of day, and standard deviations were wide. Validation analyses showed that classic wanderers had the most-severe cognitive impairment, greatest independence in mobility, and poorest general health. Although their overall health was not substantially different from some other groups, they had more-severe heart problems than other wanderers and poorer gastrointestinal and nutritional status than Cluster B (but similar to Cluster C). Nonsignificance of circadian rhythm parameters does not explain the temporal distribution of wandering for Cluster A or for any group studied; other factors, perhaps fatigue or stamina, account for earlier daytime wandering of classic wanderers. Classic wanderers differed from nonwanderers in similar ways, having significantly more-severe cognitive impairment and better mobility, more-severe heart problems, slightly less-severe problems with the upper gastrointestinal tract and nutritional status, and similar problems in the lower gastrointestinal tract. Nursing staff were most likely to identify classic wanderers as wanderers.

Members of Cluster B, or moderate wanderers, had appreciably lower wandering rates and durations than classic wanderers. They wandered in approximately half of observations and less within observation periods than classic wanderers. Validation analyses showed that moderate wanderers had less cognitive impairment than classic wanderers (but more than Cluster C), poorer independence in mobility (but similar to Cluster C), and the most robust health of all wanderers, overall and in specific systems. Moderate wanderers had significantly better upper and lower gastrointestinal health than nonwanderers and a similar level of cognitive impairment, slightly better mobility, and better health overall. Nursing staff were also highly likely to identify moderate wanderers as wanderers.

Cluster C, or subclinical wanderers, demonstrated low levels of wandering sporadically during the day and at levels that may be too low for nursing staff to notice or to warrant classification, with nursing staff misclassifying more than half of subclinical wanderers as nonwanderers. Validation analyses indicated that subclinical wanderers had the least cognitive impairment of all wanderers, a level of mobility independence similar to that of moderate wanderers, and slightly fewer heart and lower gastrointestinal problems than classic wanderers but poorer overall and selected aspects of health than moderate wanderers. Subclinical wanderers had less cognitive impairment, better mobility, and poorer health than nonwanderers, although these predictors were not significant individually in the multinomial regression.

\section{Implications}

Although three distinct clusters of wanderers were found, variance within groups was high on many wandering parameters. Thus, all observed parameters may not serve equally well to distinguish cluster membership from clinical observations of wandering. Overall duration and proportion of hours with any wandering may be the best indicators, because overlap across clusters was least for these parameters. Other factors, such as degree of cognitive impairment, general and specific health problems, and mobility, help distinguish between clusters of wanderers and between wanderers and nonwanderers. These factors may reflect lower cognitive capacities associated with dementia, such as for wayfinding or executive functioning, or poor cognitive capacity secondary to cardiac insufficiencies affecting brain perfusion, thereby increasing wandering, as may be the case for classic wanderers, or factors may limit capacity to wander, such as greater mobility problems, illness-related limitations (e.g., pain or discomfort secondary to gastrointestinal problem), or low energy secondary to nutritional or cardiac conditions, as may explain subclinical wanderers and nonwanderers. These interpretations are consistent with the NDB model, ${ }^{16}$ which postulates these characteristics as risk factors for wandering.

Although further examination of specific cardiac, gastrointestinal, and nutritional problems; functional mobility impairments, such as gait and balance; and cognitive deficits, such as attention and wayfinding, are needed to evaluate these interpretations, results point toward several avenues of intervention. When the amount of wandering is problematic, as often occurs for classic wanderers, treatments that optimize cardiac functioning may reduce wandering and, through possible effects on brain perfusion and cognition, may improve functioning in other areas. This suggestion is counter to prevailing beliefs that wandering is a benign behavior so long as exit control is sustained. It is also consistent with an early report about wandering as a phenomenon occurring with cardiac decompensation. ${ }^{34}$ Clinicians should also be alert for persons with dementia who fit the profile of subclinical wanderers, because their wandering often goes unrecognized. For subclinical and nonwanderers, low levels of locomotor output, including wandering, may be artificially so, and health may be improved by provision of suitable mobility supports or rehabilitation, relief of pain and discomfort, and improved nutrition. Although there is risk that wandering may increase in these groups when so treated, benefits to overall health may warrant the risk.

Results of this study also point to the importance of careful measurement in future studies examining wandering. Wandering is a multifactorial problem not solely explained by degree of cognitive impairment. Mere classification of 
individuals as wanderers or not is an insufficiently sensitive measurement approach.

\section{Limitations}

A limitation of this study is its use of only daytime (8:00 a.m. to 8:00 p.m.) wandering parameters. Although circadian rhythm was evaluated using around-the-clock body temperature data, practical considerations limited observations of wandering to daytime hours. Continuous measurement of wandering would strengthen future studies, especially those evaluating circadian disturbances in wanderers.

The group of nonwanderers in this study was formed according to the absence of observed wandering. These individuals may have wandered at times other than those observed, including nighttime. Having the poorest mobility and oldest age and being among those with the poorest health and lowest likelihood of identification as wanderers by nursing staff suggest that they may constitute a distinct group (nonwanderers or, at most, individuals with dementia who are considered ambulatory but have the least capacity or tendency to wander).

\section{CONCLUSION}

This study revealed three types of wanderers (classic, moderate, and subclinical), distinguished primarily by duration and rate of their wandering behavior. Cluster validation revealed differences between clusters and in comparison with nonwanderers in degree of cognitive impairment, mobility, and health indices. Measures of circadian rhythm, age, and sex did not differentiate types. Nursing staff classification as wanderers or nonwanderers was significantly associated with cluster membership, although wanderers with higher levels of wandering (classic and moderate) were correctly classified most often.

\section{ACKNOWLEDGMENTS}

Conflict of Interest: The editor in chief has reviewed the conflict of interest checklist provided by the authors and has determined that the authors have no financial or any other kind of personal conflicts with this paper.

This study was supported by the National Institutes of Nursing and Aging (NR04569, Principal Investigator (PI): D. Algase) and was one in a trio of interactive research project grants to test the NDB model (NR04586, PI: A. Whall and NR04570, PI: C. Beck). The authors acknowledge the contributions of Dr. Jun-Ah Song, who assisted with data collection and coding; Dr. Gwi-Ryung Son Hong and Mr. Barry DeCicco, who contributed to data management; and members of the NDB Interactive Research Project Group, who contributed to design and implementation plans for the parent study.

Author Contributions: Donna Algase provided leadership in the conception and design of the study and in the interpretation of data and manuscript preparation. Cathy Anatonakos planned and conducted all analyses with input from other authors and drafted relevant sections of the manuscript. Drs. Beattie and Beel-Bates were responsible for subject enrollment and oversight of data collection activities; they also contributed to interpretation of the data and drafted portions of the methods section of the manuscript. Dr. Yao contributed to data analysis and interpretation.

Sponsor's Role: The sponsor had no role in determining the design, methods, subject recruitment, data collections, analysis, or preparation of the manuscript.

\section{REFERENCES}

1. Bowen JD, Malter AD, Sheppard L et al. Predictors of mortality in patients diagnosed with probable Alzheimer's disease. Neurology 1996; 19:433-439.

2. Buchner DM, Larson EB. Falls and fractures in patients with Alzheimer-type dementia. JAMA 1987;257:1492-1495.

3. Tinetti ME, Liu WL, Marottoli RA et al. Mechanical restraint use among residents of skilled nursing facilities: Prevalence, patterns, and predictors. JAMA 1991;265:468-471.

4. Rowe M, Bennet V. A look at deaths occurring in persons with dementia lost in the community. Am J Alzheimers Dis Other Demen 2003;18:343-348.

5. Walsh JS, Welch HG, Larson EB. Survival of outpatients with Alzheimer-type dementia. Ann Intern Med 1990;113:429-434.

6. Balesteri L, Grossberg A, Grossberg GT. Behavioral and psychological symptoms of dementia as a risk factor for nursing home placement. Int Psychogeriatr 2000;3:363-366.

7. Bartels SJ, Clark RE, Peacock WJ et al. Medicare and Medicaid Costs for schizophrenia patients by age cohort compared with costs for depression, dementia, and medically ill patients. Am J Geriatr Psychiatry 2003;11:648657.

8. Foxwell LG. Elopement - exposure and control. J Long Term Care Admin 1994;21:9-12.

9. Algase DL, Antonakos C, Beattie ERA et al. New parameters for daytime wandering. Res Gerontol Nurs 2009;2:58-68.

10. Butler JP, Barnett CA. Window of wandering. Geriatr Nurs 1991;12:226-227.

11. Hirst ST, Metcalf BJ. Whys and whats of wandering. Geriatr Nurs 1989;10:237-238.

12. Hussian RA, Davis R.Analysis of wandering behavior in institutionalized geriatric patients. Presented at meeting of the Association for Behavioral Analysis, Milwaukee, WI, 1983.

13. Martino-Saltzman D, Blasch BB, Morris RD et al. Travel behavior of nursing home residents perceived as wanderers and nonwanderers. Gerontologist 1991;31:666-672. UI: 1778493.

14. Algase DL, Kupferschmid B, Beel-Bates CA et al. Estimates of stability of daily wandering behavior among cognitively impaired long-term care residents. Nurs Res 1007;46:172-178.

15. Algase DL, Beattie ER, Therrien B. Impact of cognitive impairment on wandering behavior. West J Nurs Res 2001;23:283-295. UI: 11291432.

16. Algase DL, Beck C, Kolanowski A et al. Need-driven dementia-compromised behavior: An alternative view of disruptive behavior. Am J Alzheimers Dis Other Demen 1996;11:10-19.

17. Algase DL, Antonakos C, Yao L et al. Are wandering and physically nonaggressive agitation equivalent? Am J Geriatr Psychiatry 2008;16:293299.

18. Algase DL, Beattie ERA, Bogue EL et al. The Algase Wandering Scale: Initial psychometrics of a new caregiver reporting tool. Am J Alzheimers Dis Other Demen 2001;16:141-152. UI: 11398562.

19. Folstein MF, Folstein SE, McHugh PR. Mini-mental state: A practical method for grading the cognitive state of patients for the clinician. J Psychiatr Res 1975;12:189-198.

20. Folstein MF, McHugh PR. Psychopathology of dementia: Implications for neuropathology. In: Katzman R, editor. Congenital and Acquired Cognitive Disorders. New York: Raven Press, 1979, pp 189-195.

21. Tsai L, Tsuang M. The mini-mental state test and computerized tomography. Am J Psychiatry 1979;23:433-441.

22. Song J, Algase DL, Beattie ERA et al. Comparison of U.S., Canadian and Australian participants' performance on the Algase Wandering Scale-version 2 (AWS-V2). Res Theory Nurs Pract 2003;17:241-256.

23. Yao L, Algase D. Environmental ambiance as a new window on wandering. West J of Nurs Res 2006;28:89-104.

24. Hawes C, Morris J, Phillips CD et al. Development of the nursing home resident assessment in the USA. Age Ageing 1997;26(Suppl. 2):19-26. UI: 9464550.

25. Hawes C, Morris JN, Phillips CD et al. Reliability estimates for the Minimum Data Set for nursing home resident assessment and care screening (MDS). Gerontologist 1995;35:172-178. UI: 7750773. 
26. Phillips CD, Morris JN. The potential use of administrative and clinical data to analyze outcomes for the cognitively impaired: Evaluating the Minimum Data Set for nursing homes. Alzheimer Dis Assoc Disord 1995;11(Suppl 6):162167.

27. Miller MD, Paradis CF, Houck PR et al. Rating chronic medical illness burden in geropsychiatric practice and research: Application of the Cumulative Illness Rating Scale. Psychiatry Res 1992;41:237-248.

28. Schnelle JF, Ouslander JG, Simmons SF etal. The nighttime environment, incontinence care, and sleep disruption in nursing homes. J Am Geriatr Soc 1993;41:910-4. UI: 8409176.

29. Schmitz T, Bair N, Falk M et al. A comparison of five methods of temperature measurement in febrile intensive care patients. Am J Crit Care 1995;4:286-92. UI: 7663592.
30. Lattavo K, Britt J, Dobal M. Agreement between measures of pulmonary artery and tympanic temperatures. Res Nurs Health 1995;18:365-70. UI: 7624530 .

31. Erickson RS, Meyer LT. Accuracy of infrared ear thermometry and other temperature methods in adults. Am J Crit Care 1994;3:40-54. UI: 8118492 .

32. Marler MR, Gehrman P, Martin JL et al. The sigmoidally transformed cosine curve: A mathematical model for circadian rhythms with symmetric non-sinusoidal shapes. Stat Med 2006;25:3893-3904. UI: 16381069.

33. Cohen-Mansfield J, Werner P, Marx MS et al. Two studies of pacing in the nursing home. J Gerontol 1991;46:M77-83. UI: 1992956.

34. Soverini S, Borghesi E. On a strange case of wandering in an arteriosclerotic demented patient. J Gerontol 1968;16:846-851. 